\title{
EFFECT OF ORGANIC AND BIO-FERTILIZER SOURCES ON SUMMER SQUASH UNDER EL-ARISH CONDITIONS
}

\author{
Saad K. Hafez ${ }^{1}$, A.K. El-Beik ${ }^{2}$, E.M. El-Tantawy ${ }^{2}$ and A.I. El-Kassas ${ }^{* 2}$ \\ 1. Center Agric. Res., Min. Agric. \& Land Reclam., Egypt. \\ 2. Dept. Plant Prod., Fac. Environ. Agric. Sci., Arish Univ., Egypt.
}

\begin{abstract}
A field study was carried out during summer seasons of 2013 and 2014 at the Experimental Farm, Faculty of Environmental Agricultural Sciences, Arish University, Egypt. It aims to study the effect of organic fertilizer sources (cow manure "CM"; chicken manure (ChM); pressed olive cake (POC); compost "Comp" $\}$ and two bio-fertilizer sources (Bio-1 and Bio-2) plus without biofertilizer on summer squash (Cucurbita pepo L.) $c v$ "Askandrany" under ELArish conditions. The experiment included 12 treatments and the experimental design was split plot design with three replications. Organic fertilizers were randomly distributed in the main plots (four sources), while, sub plots contained three bio treatments (without bio, Bio-1 and Bio-2). The obtained results indicated that cow manure treatment gave the highest values of number of fruits/plant, mean fruit weight, yield/plant and yield/fed., also, cow manure $\mathrm{x}$ Bio-1 treatment gave the highest value in case of average fruit weight/plant, while, cow manure $\mathrm{x}$ Bio-2 treatment gave the superior values in cases of number of fruits/plant, yield/ plant and yield/fed., in both seasons. Results of the interaction between organic and bio fertilizers, chicken manure and Bio-1 produced the highest value of TSS\%.
\end{abstract}

Kew words: Organic, bio-fertilizer, cow manure "CM", chicken manure (ChM), pressed olive cake (POC), compost "Comp", summer squash.

\section{INTRODUCTION}

Squash (Cucurbita pepo L.) is one of the most popular vegetable crops in Egypt. It has a high vital nature due to its high content of vitamins A, B3 (niacin) and moderate content of vitamin $\mathrm{C}$ and riboflavin. The total cultivated area of squash in Egypt reached about 33213 feddans in 2013 season, which produced about 262102 tons with an average yield of 7.892 ton/fed. To reduce and eliminate the adverse effects of synthetic fertilizers and pesticides on human health and environment, new agricultural practices have been developed in the so-called organic agriculture, ecological agriculture or sustainable agriculture (Aksoy, 2001).

\footnotetext{
* Corresponding author: Tel.: + 01221702119

E-mail address: alielkasas_elkasas@yahoo.com
}

Marketing organic crops in Egypt is mainly produced for exports. Local consumption of organic vegetables and fruits followed in latter stage, but of a lower significance and requires large efforts to be organized (Abo-Hadid, 2001). Excessive use of chemical fertilizers adversely affects the soil environment leading to declining crop productivity and production of potentially harmful food, unsafe for human consumption, these unintended harmful effects and the energy intensive processes have resulted in several research initiatives for developing organic fertilizer alternatives (Chaturvedi et al., 2012) because use organic matter such as animal manures, human wastes, food wastes, yard wastes, sewage sludge and compost has long been recognized in agriculture as beneficial for 
plant growth and yield and improving soil structure, enhancing soil fertility and increasing crop yield.

Biofertilizer is a natural product carrying living microorganisms derived from the root or cultivated soil, so they don't have any effect on soil health and environment, besides their role in atmospheric nitrogen fixation and phosphorous solubolization, these also help in stimulating the plant growth hormones providing better nutrient uptake, a small dose of biofertilizer is sufficient to produce desirable results because each gram of carrier of bio fertilizers contains at least 10 million viable cells of a specific strain; i.e., Azotobacter, Azospirillum and biogein (Ramakrishnan and Selvakumar, 2012).

Therefore, it is essential to adopt a system of organic farming in vegetables due to increasing the objectives against the chemical farming as a main source of soil and water pollution as well as food products. Organic farming is a system that excludes the use of synthetic fertilizers, pesticides and growth regulators. Insects, weeds and other pests are managed by mechanical cultivation, cultural and biological control.

Cow manure, chicken manure, pressed olive cake and compost are good sources of organic fertilizers. These sources play an important role in increasing growth, yield and yield components of many crops. The objective of this study was to study the effect of organic fertilizers; viz., cow manure "CM", chicken manure (ChM), pressed olive cake (POC) and compost "Comp" on the productivity of summer squash in order to produce high yield and fruit quality with less contamination.

\section{MATERIALS AND METHODS}

A field study was carried out during summer seasons of 2013 and 2014 at the Experimental Farm, Faculty of Environmental Agricultural Sciences, Arish
University. It aims to study the effect of organic fertilizer sources [cow manure $(\mathrm{CM})$; chicken manure (ChM); pressed olive cake (POC); compost (Comp) and two bio-fertilizer sources (Bio-1 and Bio-2)] on summer squash (Cucurbita pepoL.) cv. "Askandrany" under El-Arish conditions. Bio-1, is one of the beneficial bacteria (Frateuria aurentia, family Psudomonaceae) in potassium edit facilitator of absorbed share by the plant in all soil types, while, Bio-2, is a biological enriched vital (powder) containing a combination of types of fungi mikroheeza and another set of micro-organisms living in the soil, such as bacteria (Bacillus megaterium) which plays a vital important role in facilitating the phosphorus in the soil. The physical and chemical analyses of the soil and irrigation water as well as analyses of organic fertilizers is presented in Tables 1, 2, 3 and 4 , respectively.

The experiment included 12 treatments as follows: Control + without Bio (cow manure), Control + Bio-1, Control + Bio-2, Chicken manure + without Bio, Chicken manure + Bio-1, Chicken manure + Bio-2, Pressed olive cake + without Bio, Pressed olive cake + Bio-1, Pressed olive cake + Bio-2, Compost + without Bio, Compost + Bio-1, and Compost+Bio-2. The experimental design was split plot design with three replications. Organic fertilizers were randomly distributed in the main plots (four sources), while, sub plots contained three bio treatments (without bio, Bio-1 and Bio2). The sub - plot area was $10 \mathrm{~m}^{2}(1 \mathrm{~m}$ width $\mathrm{x} 10 \mathrm{~m}$ in length, and $50 \mathrm{~cm}$ spacing between plants in the raw). The amounts of organic fertilizers were calculated based on percent in the cow manure. Organic manures were added at the time of soil preparation, trenched in the bottom of the row and covered by $20 \mathrm{~cm}$ height of soil. Summer squash seeds were sown on April $8^{\text {th }} 2013$ and 2014, agricultural practices were applied according the organic farming recommendations. 
SINAI Journal of Applied Sciences (ISSN: 2314-6079) Vol. (5) Is. (2), Aug. 2016

Table (1): The physical and chemical analyses of the experimental soil site.

\begin{tabular}{|c|c|c|}
\hline \multirow{3}{*}{ Soil properties } & First season(2013) & Second season (2014) \\
\hline & \multicolumn{2}{|c|}{ Soil depth (cm.) } \\
\hline & \multicolumn{2}{|c|}{ 0-30 } \\
\hline \multicolumn{3}{|c|}{ Mechanical analysis } \\
\hline Coarse sand (\%) & 68.00 & 67.99 \\
\hline Fine sand $(\%)$ & 20.60 & 20.55 \\
\hline Silt (\%) & 3.50 & 3.52 \\
\hline Clay $(\%)$ & 7.90 & 7.94 \\
\hline Soil texture class & Sandy & Sandy \\
\hline Bulk density $\left({\left.\mathrm{g} . \mathrm{cm}^{-3}\right)}^{-3}\right.$ & 1.53 & 1.53 \\
\hline Particle density $\left(\mathrm{g} \cdot \mathrm{cm}^{-3}\right)$ & 2.49 & 2.49 \\
\hline \multicolumn{3}{|c|}{ Chemical analysis (soluble ions in (1:5) extract) } \\
\hline $\mathrm{Ca}^{++}\left(\right.$meq..$\left.^{-1}\right)$ & 3.03 & 2.10 \\
\hline $\operatorname{Mg}^{++}\left(\operatorname{meq.1}{ }^{-1}\right)$ & 2.11 & 2.20 \\
\hline $\mathrm{Na}^{+}\left(\right.$meq. $\left.\mathbf{1}^{-1}\right)$ & 1.18 & 4.49 \\
\hline $\mathrm{K}^{+}\left(\mathbf{m e q} . \mathbf{1}^{-1}\right)$ & 0.48 & 0.31 \\
\hline $\mathrm{CO}_{3}^{--}\left(\operatorname{meq} .1^{-1}\right)$ & - & - \\
\hline $\mathrm{HCO}_{3}^{-}\left(\operatorname{meq} .1^{-1}\right)$ & 2.00 & 2.40 \\
\hline $\mathrm{Cl}^{-}\left(\right.$meq. $\left.\mathbf{1}^{-1}\right)$ & 1.02 & 2.30 \\
\hline $\mathrm{SO}^{--}\left(\operatorname{meq} .1^{-1}\right)$ & 3.78 & 4.40 \\
\hline EC $\left(\mathrm{dS} \mathrm{m}^{-1}\right)$ (soil paste) & 0.68 & 0.91 \\
\hline pH in (1:2.5 extract) & 8.10 & 8.20 \\
\hline Organic matter $(\%)$ in air dried soil & 0.16 & 0.21 \\
\hline $\mathrm{CaCO}_{3}(\%)$ in air dried soil & 3.95 & 3.95 \\
\hline
\end{tabular}

Table (2): The physical and chemical analyses of irrigation water.

\begin{tabular}{|c|c|c|c|c|c|c|c|c|}
\hline \multirow[t]{2}{*}{ EC } & \multicolumn{8}{|c|}{ Soluble ions $\left(\right.$ meq. $\left.^{-1}\right)$} \\
\hline & \multicolumn{3}{|c|}{ Cations } & \multicolumn{5}{|c|}{ Anions } \\
\hline $\mathrm{dS} \mathrm{m}^{-1}$ & $\mathbf{C a}^{++}$ & $\mathbf{M g}^{++}$ & $\mathrm{Na}^{+}$ & $\mathbf{K}^{+}$ & $\mathrm{Cl}^{-}$ & $\mathrm{HCO}_{3}^{-}$ & $\mathrm{CO}_{3}^{-}$ & $\mathrm{SO}_{4}^{--}$ \\
\hline 7.03 & 16.56 & 17.60 & 35.87 & 0.27 & 42.26 & 6.13 & - & 21.91 \\
\hline
\end{tabular}


Table (3): Analyses of organic fertilizer compost.

\begin{tabular}{lcc}
\hline \multicolumn{1}{c}{$\begin{array}{c}\text { Organic fertilizer } \\
\text { Contents }\end{array}$} & $\begin{array}{c}\text { First season } \\
(\mathbf{2 0 1 3})\end{array}$ & $\begin{array}{c}\text { Second season } \\
(\mathbf{2 0 1 4})\end{array}$ \\
\hline Total N (\%) & 0.71 & 0.69 \\
Total P (\%) & 0.39 & 0.49 \\
Total K (\%) & 0.42 & 0.29 \\
Total Fe (ppm) & 1110 & 1188 \\
Total Cu (ppm) & 123 & 110 \\
Total Zn (ppm) & 219 & 214 \\
Total Mn (ppm) & 170 & 166 \\
Organic matter (\%) & 30.20 & 24.05 \\
Organic carbon (\%) & 12.88 & 13.95 \\
C/N (\%) & 18.14 & 20.20 \\
\hline
\end{tabular}

Source: Center laboratory of Organic Agriculture, Agri. Res. Center, Ministry of Agriculture.

Table (4): Chemical analyses of organic fertilizers.

\begin{tabular}{cccc}
\hline \multirow{2}{*}{$\begin{array}{c}\text { Organic fertilizer } \\
\text { contents }\end{array}$} & \multicolumn{3}{c}{ Organic Fertilizers } \\
\cline { 2 - 4 } & Cow manure & Chicken manure & Pressed Olive Cake \\
\hline & \multicolumn{2}{c}{ First season (2013) } \\
Total N (\%) & 2.8 & 2.1 & 2.1 \\
Total P (\%) & 0.3 & 0.1 & 0.3 \\
Total K (\%) & 2.0 & 1.0 & 2.0 \\
& \multicolumn{2}{c}{ Second season (2014) } \\
Total N (\%) & 6.0 & 1.1 & 1.2 \\
Total P (\%) & 0.3 & 0.3 & 0.3 \\
Total K (\%) & 1.7 & 1.5 & 2.0 \\
\hline
\end{tabular}

\section{Data Recorded}

\section{Vegetative growth characters}

Random samples of Three summer squash plants of every replicate were taken at 20 and 40 days after sowing (DAS) in both seasons for conducting the following growth parameters: stem length $(\mathrm{cm})$, number of leaves per plant, leaf area/plant $(\mathrm{cm})$, and total dry weight /plant.

\section{Flowering characters}

A random sample of five plants were taken from every treatment during both studying seasons to calculate the percent of female flowers, then sexratio was determined by dividing number of staminate flowers by number of pistillate flowers.

\section{Yield and its components}

Fruits were harvested day after day up to the end of plant production; productivity was determined as number of fruits/plant, yield/plant (g) and total fruit yield (ton/fed.)

\section{Fruit quality}

Total soluble solids percentage (TSS \%) was determined in the extract of fresh fruits 
by using a hand refractometer. Fruit diameter and fruit length $(\mathrm{cm})$ at commercial stage were determined using a Caliper. Firmness $\left(\mathrm{Kg} / \mathrm{cm}^{2}\right)$ was determined by using hand firmness tester Model ST207 , made in Italy.

\section{Plant chemical composition}

Total nitrogen, phosphorus and potassium were determined in dry matter of leaves from the third sample (at 60 days) methods described by Bremner and Mulvaney (1982), Piper 1950, and Brown and Lilliand (1946), respectively.

Data subjected to statistical analysis as randomized complete block design in a split plot after planting) and from the second harvest (at 80 days after transplanting) using the with three replicates in both seasons. MstatC program was used for statistical analysis, and data were tested by analysis of variance. Duncan's multiple range test was used for comparison among the treatment means (Duncan, 1958).

\section{RESULTS AND DISCUSSION}

\section{Vegetative Growth Characters}

\section{Effect of organic fertilizers}

Results in Tables 5 and 6 clear that organic fertilizer sources affected significantly stem length, number of leaves, leaf area and total dry weight per pant after twenty and forty days after sowing in both seasons, except, number of leaves at 20 days after sowing in the first season. Compost treatment resulted in the highest value for each of stem length, number of leaves and leaf area at 20 days after sowing, while cow manure gave the highest values at 40 days after sowing. However, application of cow manure or chicken manure gave the highest values for total dry weight at 20 and 40 days after sowing in both seasons.

The superiority of compost at 20 days after sowing may be due to the enough time allowed for compost analysis before soil application as compared with other organic sources obtained from organic forms in incomplete analysis to confine mineralization after soil application. The increment in dry weight of summer squash plant may be due to the simulative effect of organic fertilizer treatments on the meristematic activity of tissues, where these treatments contained adequate nutrients required for plant growth as reported by Safia et al. (2001).

Also, results may be due to the effect of organic fertilizers on soil physiochemical and biological properties as reported by Darwesh, Faysa (2002). In addition, our results are in agreement with those reported by El-Ghanam et al. (2005) who found that organic fertilizer application caused a reduction in soil $\mathrm{pH}$ in the rhizosphere zone which may be due to the formation of $\mathrm{CO}_{2}$ and other organic acids during decomposition of the organic fertilizer.

\section{Effect of bio fertilizers}

Results in Tables 5 and 6 clear that biofertilizers had no significant effect on most of vegetative growth characters, except for stem length at 20 days after sowing and leaf area 40 days after sowing in the second season, which increased significantly with application of Bio-2 and Bio-1, respectively.

\section{Effect of interaction between organic fertilizers and Bio fertilizers}

Results in Tables 5 and 6 show that the interaction between organic fertilizers and bio-fertilizers affected significantly most vegetative characters, except, number of leaves/plant at 20 days in the second season.

The increases in most vegetative growth traits may be refer to the ability of biofertilizers to release some chemical compounds that may affect the improvement of plant growth characters, it is interest to note that Organic fertilizer contributes through: (a) release of nutrients through the decomposition of organic matter 
Table (5): Effect of organic fertilizers, bio-fertilizers and their interactions on vegetative growth characters of summer squash during 2013 season.

\begin{tabular}{|c|c|c|c|c|c|c|c|c|}
\hline \multirow{3}{*}{ Treatment } & \multicolumn{2}{|c|}{$\begin{array}{l}\text { Stem length } \\
(\mathrm{cm})\end{array}$} & \multicolumn{2}{|c|}{$\begin{array}{c}\text { No. leaves/ } \\
\text { plant }\end{array}$} & \multicolumn{2}{|c|}{$\begin{array}{l}\text { leaf area/plant } \\
\left(\mathrm{cm}^{2}\right)\end{array}$} & \multicolumn{2}{|c|}{$\begin{array}{l}\text { Plant dry weight } \\
\text { (g) }\end{array}$} \\
\hline & \multicolumn{8}{|c|}{ Days after sowing } \\
\hline & 20 & 40 & 20 & 40 & 20 & 40 & 20 & 40 \\
\hline \multicolumn{9}{|c|}{ Organic fertilizers First season (2013) } \\
\hline Cow manure & $2.68 b$ & $10.22 \mathrm{a}$ & $5.61 \mathrm{a}$ & $12.56 \mathrm{a}$ & $205.7 b$ & $2636.0 \mathrm{a}$ & $4.34 \mathrm{a}$ & $25.99 \mathrm{ab}$ \\
\hline Chicken manure & $2.86 \mathrm{~b}$ & $9.39 \mathrm{a}$ & $6.06 \mathrm{a}$ & $12.61 \mathrm{a}$ & $204.4 b$ & $2658.0 \mathrm{a}$ & $4.26 \mathrm{ab}$ & $28.72 \mathrm{a}$ \\
\hline Pressed olive cake & $3.56 \mathrm{a}$ & $5.53 b$ & $6.0 \mathrm{a}$ & $8.33 c$ & $156.6 \mathrm{c}$ & $634.20 \mathrm{c}$ & $1.84 \mathrm{c}$ & $18.29 b$ \\
\hline Compost & $3.83 a$ & $7.08 \mathrm{~b}$ & $6.72 \mathrm{a}$ & $10.0 \mathrm{~b}$ & $258.4 \mathrm{a}$ & $1352.0 \mathrm{~b}$ & $3.63 b$ & $20.77 \mathrm{ab}$ \\
\hline \multicolumn{9}{|l|}{ Bio fertilizers } \\
\hline Without bio & $3.04 \mathrm{a}$ & $7.73 \mathrm{a}$ & $6.13 \mathrm{a}$ & $10.92 \mathrm{a}$ & $202.1 \mathrm{a}$ & $1820.0 \mathrm{a}$ & $3.33 \mathrm{a}$ & $23.22 \mathrm{a}$ \\
\hline Bio-1 & $3.15 \mathrm{a}$ & $8.25 \mathrm{a}$ & $6.0 \mathrm{a}$ & $11.17 \mathrm{a}$ & $202.0 \mathrm{a}$ & $1959.0 \mathrm{a}$ & $3.66 \mathrm{a}$ & $23.33 \mathrm{a}$ \\
\hline Bio- 2 & $3.50 \mathrm{a}$ & $8.19 \mathrm{a}$ & $6.17 \mathrm{a}$ & $10.54 \mathrm{a}$ & $214.8 \mathrm{a}$ & $1682.0 \mathrm{a}$ & $3.55 \mathrm{a}$ & $23.78 \mathrm{a}$ \\
\hline \multicolumn{9}{|l|}{ Organic fertilizers $x$ bio fertilizers } \\
\hline Cow manure $x$ without bio & $2.42 \mathrm{~d}$ & $9.83 \mathrm{ab}$ & $5.17 \mathrm{c}$ & $12.0 \mathrm{a}$ & $192.8 \mathrm{~cd}$ & $2363.0 \mathrm{~cd}$ & $3.98 \mathrm{bc}$ & $24.03 \mathrm{a}-\mathrm{c}$ \\
\hline Cow manure $x$ bio-1 & $2.53 \mathrm{~cd}$ & $11.0 \mathrm{a}$ & $5.67 \mathrm{bc}$ & $13.33 \mathrm{a}$ & $211.2 \mathrm{~cd}$ & $2995.0 \mathrm{a}$ & $4.77 \mathrm{a}$ & $26.73 \mathrm{ab}$ \\
\hline Cow manure $x$ bio-2 & $3.08 \mathrm{bc}$ & $9.83 \mathrm{ab}$ & $6.0 \mathrm{bc}$ & $12.33 \mathrm{a}$ & $213.1 \mathrm{~cd}$ & $2549.0 \mathrm{bc}$ & $4.27 \mathrm{a}-\mathrm{c}$ & $27.22 \mathrm{ab}$ \\
\hline Chicken manure $x$ without bio & $2.75 \mathrm{~cd}$ & $8.83 \mathrm{a}-\mathrm{c}$ & $5.83 \mathrm{bc}$ & $13.17 \mathrm{a}$ & $202.6 \mathrm{~cd}$ & $3009.0 \mathrm{a}$ & $4.63 \mathrm{ab}$ & $29.90 \mathrm{a}$ \\
\hline Chicken manure $x$ bio-1 & $2.75 \mathrm{~cd}$ & $10.33 \mathrm{ab}$ & $6.0 \mathrm{bc}$ & $12.83 \mathrm{a}$ & $186.3 \mathrm{de}$ & $2843.0 \mathrm{ab}$ & $3.85 \mathrm{c}$ & $27.17 \mathrm{ab}$ \\
\hline Chicken manure $x$ bio-2 & $3.08 \mathrm{bc}$ & $9.0 \mathrm{a}-\mathrm{c}$ & $6.33 \mathrm{a}-\mathrm{c}$ & $11.83 \mathrm{ab}$ & $224.3 \mathrm{bc}$ & $2123.0 \mathrm{~d}$ & $4.30 \mathrm{a}-\mathrm{c}$ & $29.08 \mathrm{a}$ \\
\hline Pressed olive cake $x$ without bio & $3.08 \mathrm{bc}$ & $6.17 \mathrm{de}$ & $6.33 \mathrm{a}-\mathrm{c}$ & $8.17 \mathrm{~d}$ & $151.8 \mathrm{f}$ & $632.5 f$ & $1.73 \mathrm{e}$ & $19.07 \mathrm{c}$ \\
\hline Pressed Olive Cake x Bio-1 & $3.75 \mathrm{a}$ & $4.5 \mathrm{e}$ & $5.83 \mathrm{bc}$ & $8.67 \mathrm{~cd}$ & $157.7 \mathrm{ef}$ & $652.7 f$ & $1.95 \mathrm{e}$ & $18.12 \mathrm{c}$ \\
\hline Pressed Olive Cake x Bio-2 & $3.83 \mathrm{a}$ & $5.92 \mathrm{de}$ & $5.83 \mathrm{bc}$ & $8.17 \mathrm{~d}$ & $160.3 \mathrm{ef}$ & $617.3 \mathrm{f}$ & $1.83 \mathrm{e}$ & $17.70 \mathrm{c}$ \\
\hline Compost x Without Bio & $3.92 \mathrm{a}$ & $6.08 \mathrm{de}$ & $7.17 \mathrm{a}$ & $10.33 \mathrm{bc}$ & $261.1 \mathrm{a}$ & $1274.0 \mathrm{e}$ & $2.98 \mathrm{~d}$ & $19.88 b c$ \\
\hline Compost x Bio-1 & $3.58 \mathrm{ab}$ & $7.17 \mathrm{~cd}$ & $6.5 \mathrm{ab}$ & $9.83 \mathrm{~cd}$ & $252.9 \mathrm{ab}$ & $1343.0 \mathrm{e}$ & $4.07 \mathrm{a}-\mathrm{c}$ & $21.32 \mathrm{bc}$ \\
\hline Compost x Bio-2 & $4.0 \mathrm{a}$ & $8.0 \mathrm{~b}-\mathrm{d}$ & $6.5 \mathrm{ab}$ & $9.83 \mathrm{~cd}$ & $261.3 \mathrm{a}$ & $1437.0 \mathrm{e}$ & $3.82 \mathrm{c}$ & $21.12 b c$ \\
\hline
\end{tabular}


SINAI Journal of Applied Sciences (ISSN: 2314-6079) Vol. (5) Is.:(1), Aug. 2016

Table (6): Effect of organic fertilizers, bio-fertilizers and their interactions on vegetative growth characters of summer squash during 2014 season.

\begin{tabular}{|c|c|c|c|c|c|c|c|c|}
\hline \multirow{3}{*}{ Treatment } & \multicolumn{2}{|c|}{ Stem length(cm) } & \multicolumn{2}{|c|}{ No. leaves/Plant } & \multicolumn{2}{|c|}{$\begin{array}{l}\text { leaf area/plant } \\
\left(\mathrm{cm}^{2}\right)\end{array}$} & \multicolumn{2}{|c|}{$\begin{array}{c}\text { Plant dry weight } \\
\text { (gm) }\end{array}$} \\
\hline & \multicolumn{8}{|c|}{ Days after sowing } \\
\hline & 20 & 40 & 20 & 40 & 20 & 40 & 20 & 40 \\
\hline \multicolumn{9}{|c|}{ Organic fertilizers Second season (2014) } \\
\hline Cow manure & $2.65 \mathrm{~b}$ & $10.08 \mathrm{a}$ & $5.64 \mathrm{~b}$ & $12.33 \mathrm{a}$ & $202.9 \mathrm{~b}$ & $2642.0 \mathrm{a}$ & $4.39 \mathrm{a}$ & $24.67 \mathrm{ab}$ \\
\hline Chicken manure & $2.89 \mathrm{~b}$ & $9.19 \mathrm{~b}$ & $6.11 \mathrm{ab}$ & $12.61 \mathrm{a}$ & $207.9 \mathrm{~b}$ & $2601.0 \mathrm{a}$ & $4.23 \mathrm{a}$ & $28.47 \mathrm{a}$ \\
\hline Pressed Olive Cake & $3.61 \mathrm{a}$ & $5.46 \mathrm{~d}$ & $6.03 \mathrm{a}$ & $8.36 \mathrm{c}$ & $155.1 \mathrm{c}$ & $631.9 \mathrm{c}$ & $1.84 \mathrm{c}$ & $18.40 \mathrm{c}$ \\
\hline Compost & $3.75 \mathrm{a}$ & $7.03 \mathrm{c}$ & $6.64 \mathrm{a}$ & $9.89 \mathrm{~b}$ & $257.5 \mathrm{a}$ & $1353.0 \mathrm{~b}$ & $3.66 \mathrm{~b}$ & $21.06 \mathrm{bc}$ \\
\hline \multicolumn{9}{|l|}{ Bio fertilizers } \\
\hline Without Bio & $3.02 \mathrm{~b}$ & $7.58 \mathrm{a}$ & $6.04 \mathrm{a}$ & $10.85 \mathrm{a}$ & 199.9 a & $1827.0 \mathrm{ab}$ & $3.37 \mathrm{a}$ & $23.08 \mathrm{a}$ \\
\hline Bio-1 & $3.12 \mathrm{~b}$ & $8.10 \mathrm{a}$ & $6.02 \mathrm{a}$ & $11.02 \mathrm{a}$ & $200.0 \mathrm{a}$ & $1956.0 \mathrm{a}$ & $3.69 \mathrm{a}$ & $22.48 \mathrm{a}$ \\
\hline Bio- 2 & $3.53 \mathrm{a}$ & $8.14 \mathrm{a}$ & $6.25 \mathrm{a}$ & $10.52 \mathrm{a}$ & $217.6 \mathrm{a}$ & $1638.0 \mathrm{~b}$ & $3.54 \mathrm{a}$ & $23.90 \mathrm{a}$ \\
\hline \multicolumn{9}{|l|}{ Organic fertilizers $x$ Bio fertilizers } \\
\hline Cow manure $x$ Without Bio & $2.38 \mathrm{e}$ & $9.42 \mathrm{bc}$ & $5.250 \mathrm{e}$ & $11.67 \mathrm{c}$ & $190.6 \mathrm{de}$ & $2377.0 \mathrm{~b}$ & $4.10 \mathrm{~b}$ & $21.78 \mathrm{~cd}$ \\
\hline Cow manure x Bio-1 & $2.49 \mathrm{de}$ & $11.08 \mathrm{a}$ & $5.583 \mathrm{c}-\mathrm{e}$ & $13.08 \mathrm{ab}$ & $203.5 \mathrm{de}$ & $3007.0 \mathrm{a}$ & $4.72 \mathrm{a}$ & $25.30 \mathrm{bc}$ \\
\hline Cow manure x Bio-2 & $3.09 \mathrm{c}$ & $9.75 \mathrm{ab}$ & $6.083 \mathrm{~b}-\mathrm{e} 1$ & $12.25 \mathrm{a}-\mathrm{c}$ & $214.7 \mathrm{~cd}$ & $2542.0 \mathrm{~b}$ & $4.35 \mathrm{ab}$ & $26.94 \mathrm{ab}$ \\
\hline Chicken manure x Without Bio & $2.79 \mathrm{~cd}$ & $9.17 \mathrm{bc}$ & $5.500 \mathrm{de}$ & $13.33 \mathrm{a}$ & $203.0 \mathrm{de}$ & $3035.0 \mathrm{a}$ & $4.68 \mathrm{a}$ & $30.74 \mathrm{a}$ \\
\hline Chicken manure x Bio-1 & $2.75 \mathrm{c}-\mathrm{e}$ & $9.58 \mathrm{a}-\mathrm{c}$ & $6.250 \mathrm{a}-\mathrm{d} 1$ & $12.42 \mathrm{a}-\mathrm{c}$ & $186.2 \mathrm{e}$ & $2825.0 \mathrm{a}$ & $3.95 \mathrm{~b}$ & $25.29 \mathrm{bc}$ \\
\hline Chicken manure x Bio-2 & $3.13 \mathrm{c}$ & $8.83 \mathrm{bc}$ & $6.583 \mathrm{ab}$ & $12.08 \mathrm{bc}$ & $234.6 \mathrm{bc}$ & $1943.0 \mathrm{c}$ & $4.05 \mathrm{~b}$ & $29.39 \mathrm{ab}$ \\
\hline Pressed Olive Cake x Without Bio & $3.13 \mathrm{c}$ & $5.75 \mathrm{ef}$ & $6.333 \mathrm{a}-\mathrm{d}$ & $8.25 \mathrm{f}$ & $149.3 \mathrm{f}$ & 648.9 e & $1.68 \mathrm{~d}$ & $19.34 \mathrm{~d}$ \\
\hline Pressed Olive Cake x Bio-1 & $3.71 \mathrm{ab}$ & $4.67 \mathrm{f}$ & $5.833 \mathrm{~b}-\mathrm{e}$ & 8.83 ef & $157.1 \mathrm{f}$ & $629.2 \mathrm{e}$ & $1.96 \mathrm{~d}$ & $18.38 \mathrm{~d}$ \\
\hline Pressed Olive Cake x Bio-2 & $4.0 \mathrm{a}$ & 5.96 ef & $5.917 \mathrm{~b}-\mathrm{e}$ & $8.0 \mathrm{f}$ & $159.0 \mathrm{f}$ & $617.5 \mathrm{e}$ & $1.89 \mathrm{~d}$ & $17.49 \mathrm{~d}$ \\
\hline Compost x Without Bio & $3.79 \mathrm{ab}$ & 6.0 ef & $7.083 \mathrm{a}$ & $10.17 \mathrm{~d}$ & $256.9 \mathrm{ab}$ & $1246.0 \mathrm{~d}$ & $3.02 \mathrm{c}$ & $20.45 \mathrm{~cd}$ \\
\hline Compost x Bio-1 & $3.54 \mathrm{~b}$ & $7.08 \mathrm{de}$ & 6.417 a-c & $9.75 \mathrm{de}$ & $253.2 \mathrm{ab}$ & $1364.0 \mathrm{~d}$ & $4.12 \mathrm{~b}$ & $20.97 \mathrm{~cd}$ \\
\hline Compost x Bio-2 & $3.92 \mathrm{ab}$ & $8.0 \mathrm{~cd}$ & $6.417 \mathrm{a}-\mathrm{c}$ & $9.75 \mathrm{de}$ & $262.3 \mathrm{a}$ & $1449.0 \mathrm{~d}$ & $3.85 \mathrm{~b}$ & $21.76 \mathrm{~cd}$ \\
\hline
\end{tabular}

* Means followed by the same alphabetical latter(s) within each column are not significantly different at $5 \%$ level, according to Duncan multiple range Test. 
(b) lowering of nutrients fixation through several mechanisms such as chelating and formation of complex relatively available for plant (c) production of humates which could exchange for absorbed anions such as $\mathrm{P}$ which should be available. In addition many researchers came to similar explanation, where they indicated that organic fertilizers may increase soil fertility which reflected on the crop production potential possibly affected by changes in soil physical and chemical properties including nutrient bio availability, soil structure, water holding capacity, cation exchange capacity, soil $\mathrm{pH}$ and microbial community and activity (Marchner, 1995; Clement and Bernal, 2006; Agbede et al., 2008; Malak et al., 2008; Ayeni et al., 2010). On the other hand, Bio fertilizers plays a vital role in plant metabolism such as their effects on constituent of proteins, enzymes, hormones, vitamins, chlorophyll and photosynthesis pigments (Reddy and Reddi, 2002).

Refai et al. (2009) found that the bacterial bio-fertilizer application promoted the crop growth by increasing root number and root length, subsequently, a significant increment in stem length, number of leaves, root system can absorb more water and nutrients from soil including the applied N. Thus, $\mathrm{N}$ lose hazards to the environment is reduce especially, in reclaimed sandy soil. In this concern, Sarhan et al. (2011) found that the biological (Azotobacter) fertilizers significantly affected the vegetative (shoot) characteristics of summer squash as compared with the control treatments.

However, Shaban, Sally (2009) found that organic nitrogen fertilizer sources affected all vegetative growth parameters of summer squash; i.e., plant length, number of leaves/plant, leaf area/plant, fresh and dry weight/plant. She found, also that the highest values were recorded with compost manure.

\section{Flowering Characters}

\section{Effect of organic fertilizers}

Results presented in Table 7 clear significant differences among organic fertilizer treatments on pistillate, staminate and sex ratio, the treatment of cow manure gave the highest pistillate number and sex ratio during the two seasons.

\section{Effect of bio fertilizers}

Results in Table 7 show that there were no significant effects for bio treatments on flowering traits in both seasons.

\section{Effect of interaction between organic fertilizers and Bio fertilizers}

Results in Table 7 show that the interaction among organic fertilizers and bio-sources affected significantly number of staminate, pistillate flowers and sex ratio. The interaction between cow manure treatment and Bio-2 treatment gave the highest number of pistillate flowers and the best sex ratio at both seasons, while pressed olive cake + bio-fertilizer treatments gave the lowest values.

Results are in harmony with Refai $\boldsymbol{e t}$ al. (2009) who found that application with biofertilizer (Azotobacter wild type strain) for squash plants that received no mineral $\mathrm{N}$ fertilizer resulted in a significant increment in number of female and male flowers.

Also, Galal et al. (2012) reported that the use of Bio-fertilization resulted in a significant increment in number of female and male flowers. In addition, Abd ElFattah and Sorial (2000), on summer squash, indicated that bio-fertilizer treatment (Halex 2) significantly enhanced the induction of female flowers, which was reflected afterward on the increase of fruit yield.

\section{Yield and its Components}

\section{Effect of organic fertilizers}

Results in Table 8 clear significant differences in yield and its components among organic fertilizer sources. Cow manure treatment gave the highest value for each of number of fruits /plant, mean fruit weight, yield /plant and yield / fed. It could 
SINAI Journal of Applied Sciences (ISSN: 2314-6079) Vol. (5) Is.:(1), Aug. 2016

Table (7): Effect of organic fertilizers, bio-fertilizers and their interactions on flowering characters of summer squash during 2013and 2014 seasons.

\begin{tabular}{|c|c|c|c|c|c|c|}
\hline \multirow{2}{*}{ Treatment } & $\begin{array}{c}\text { No. } \\
\text { Pistillate } \\
\text { flowers }\end{array}$ & $\begin{array}{c}\text { No. } \\
\text { Staminate } \\
\text { flowers }\end{array}$ & $\begin{array}{c}\text { Sex } \\
\text { ratio }\end{array}$ & $\begin{array}{c}\text { No. } \\
\text { Pistillate } \\
\text { flours }\end{array}$ & $\begin{array}{c}\text { No. } \\
\text { Staminate } \\
\text { flowers }\end{array}$ & $\begin{array}{c}\text { Sex } \\
\text { ratio }\end{array}$ \\
\hline & \multicolumn{3}{|c|}{ First season (2013) } & \multicolumn{3}{|c|}{ Second season (2014) } \\
\hline \multicolumn{7}{|l|}{ Organic fertilizers } \\
\hline Cow manure & 36.67 a & $56.78 \mathrm{a}$ & $1.570 \mathrm{a}$ & $36.33 \mathrm{a}$ & $56.22 \mathrm{ab}$ & $1.566 \mathrm{~b}$ \\
\hline Chicken manure & $32.11 \mathrm{ab}$ & $58.22 \mathrm{a}$ & $1.828 \mathrm{a}$ & $31.56 \mathrm{~b}$ & $58.44 \mathrm{a}$ & $1.864 \mathrm{a}$ \\
\hline Pressed Olive Cake & $24.78 \mathrm{c}$ & $48.0 \mathrm{~b}$ & $1.953 \mathrm{a}$ & $24.89 \mathrm{c}$ & $47.39 \mathrm{c}$ & $1.909 \mathrm{a}$ \\
\hline Compost & 25.89 bc & $50.67 \mathrm{ab}$ & $1.963 \mathrm{a}$ & $26.44 \mathrm{c}$ & $50.78 \mathrm{bc}$ & $1.924 \mathrm{a}$ \\
\hline \multicolumn{7}{|l|}{ Bio fertilizers } \\
\hline Without Bio & $29.17 \mathrm{a}$ & $53.25 \mathrm{a}$ & $1.841 \mathrm{a}$ & $29.13 \mathrm{a}$ & $52.75 \mathrm{a}$ & $1.826 \mathrm{a}$ \\
\hline Bio-1 & $29.33 \mathrm{a}$ & $54.25 \mathrm{a}$ & $1.885 \mathrm{a}$ & $29.13 \mathrm{a}$ & $54.21 \mathrm{a}$ & $1.877 \mathrm{a}$ \\
\hline Bio- 2 & $31.08 \mathrm{a}$ & $52.75 \mathrm{a}$ & $1.760 \mathrm{a}$ & $31.17 \mathrm{a}$ & $52.67 \mathrm{a}$ & $1.745 \mathrm{a}$ \\
\hline \multicolumn{7}{|l|}{ Organic fertilizers $x$ Bio fertilizers } \\
\hline Cow manure $x$ Without Bio & $33.0 \mathrm{~b}$ & $56.67 \mathrm{a}-\mathrm{c}$ & $1.723 \mathrm{a}-\mathrm{c}$ & $33.17 \mathrm{bc}$ & 54.33 a-e & $1.643 \mathrm{c}$ \\
\hline Cow manure x Bio-1 & $35.67 \mathrm{~b}$ & $58.33 \mathrm{ab}$ & $1.647 \mathrm{bc}$ & $34.67 \mathrm{~b}$ & 59.17 a-c & $1.710 \mathrm{bc}$ \\
\hline Cow manure x Bio-2 & $41.33 \mathrm{a}$ & $55.33 \mathrm{a}-\mathrm{c}$ & $1.340 \mathrm{c}$ & $41.17 \mathrm{a}$ & 55.17 a-d & $1.343 \mathrm{~d}$ \\
\hline Chicken manure $x$ Without Bio & $31.0 \mathrm{~b}-\mathrm{d}$ & $54.0 \mathrm{a}-\mathrm{c}$ & $1.747 \mathrm{a}-\mathrm{c}$ & $30.50 \mathrm{~cd}$ & $54.17 \mathrm{a}-\mathrm{e}$ & $1.777 \mathrm{a}-\mathrm{c}$ \\
\hline Chicken manure x Bio-1 & $31.33 \mathrm{bc}$ & $62.33 \mathrm{a}$ & $2.017 \mathrm{ab}$ & $30.0 \mathrm{c}-\mathrm{e}$ & $61.67 \mathrm{a}$ & $2.077 \mathrm{a}$ \\
\hline Chicken manure x Bio-2 & $34.0 \mathrm{~b}$ & $58.33 \mathrm{ab}$ & $1.720 \mathrm{a}-\mathrm{c}$ & $34.17 \mathrm{~b}$ & $59.50 \mathrm{ab}$ & $1.740 \mathrm{bc}$ \\
\hline Pressed Olive Cake x Without Bio & $27.0 \mathrm{c}-\mathrm{e}$ & $51.0 \mathrm{bc}$ & $1.893 \mathrm{ab}$ & 27.0 ef & $51.33 \mathrm{~b}-\mathrm{f}$ & $1.903 \mathrm{a}-\mathrm{c}$ \\
\hline Pressed Olive Cake x Bio-1 & $25.0 \mathrm{e}$ & $46.0 \mathrm{c}$ & $1.867 \mathrm{ab}$ & $25.50 \mathrm{f}$ & 46.17 ef & $1.813 \mathrm{a}-\mathrm{c}$ \\
\hline Pressed Olive Cake x Bio-2 & $22.33 \mathrm{e}$ & $47.0 \mathrm{c}$ & $2.100 \mathrm{a}$ & $22.17 \mathrm{~g}$ & $44.67 \mathrm{~F}$ & $2.010 \mathrm{ab}$ \\
\hline Compost $x$ Without Bio & $25.67 \mathrm{e}$ & $51.33 \mathrm{bc}$ & $2.000 \mathrm{ab}$ & $25.83 \mathrm{f}$ & $51.17 \mathrm{c}-\mathrm{f}$ & $1.980 \mathrm{ab}$ \\
\hline Compost x Bio-1 & $25.33 \mathrm{e}$ & $50.33 \mathrm{bc}$ & $2.010 \mathrm{ab}$ & $26.33 \mathrm{f}$ & $49.83 \mathrm{~d}-\mathrm{f}$ & $1.907 \mathrm{a}-\mathrm{c}$ \\
\hline Compost x Bio-2 & $26.67 \mathrm{de}$ & $50.33 \mathrm{bc}$ & $1.880 \mathrm{ab}$ & $27.17 \mathrm{~d}-\mathrm{f}$ & $51.33 \mathrm{~b}-\mathrm{f}$ & $1.887 \mathrm{a}-\mathrm{c}$ \\
\hline
\end{tabular}

* Means followed by the same alphabetical latter(s) within each column are not significantly different at $5 \%$ level, according to Duncan multiple range Test. 
Table (8): Effect of organic fertilizers, bio-fertilizers and their interactions on yield and its components of summer squash during 2013 and 2014 seasons.

\begin{tabular}{|c|c|c|c|c|c|c|c|c|}
\hline \multirow{2}{*}{ Treatment } & $\begin{array}{c}\text { No. } \\
\text { Fruits/ } \\
\text { plant }\end{array}$ & $\begin{array}{c}\text { Mean fruit } \\
\text { weight } \\
\text { (gm) }\end{array}$ & $\begin{array}{c}\text { Yield/ } \\
\text { plant } \\
\text { (g) }\end{array}$ & $\begin{array}{l}\text { Yield/ } \\
\text { fed. } \\
\text { (ton) }\end{array}$ & $\begin{array}{c}\text { No. } \\
\text { Fruits/ } \\
\text { plant }\end{array}$ & $\begin{array}{c}\begin{array}{c}\text { Mean } \\
\text { fruit } \\
\text { weight }(g)\end{array} \\
\end{array}$ & $\begin{array}{c}\text { Yield/ } \\
\text { plant (g) }\end{array}$ & $\begin{array}{l}\text { Yield/ } \\
\text { fed. } \\
\text { (ton) }\end{array}$ \\
\hline & \multicolumn{4}{|c|}{ first Season (2013) } & \multicolumn{4}{|c|}{ Second Season (2014) } \\
\hline \multicolumn{9}{|l|}{ Organic fertilizers } \\
\hline Cow manure & $8.192 \mathrm{a}$ & $111.2 \mathrm{a}$ & $908.4 \mathrm{a}$ & $7.63 \mathrm{a}$ & $8.18 \mathrm{a}$ & $111.5 \mathrm{a}$ & $910.0 \mathrm{a}$ & $7.64 \mathrm{a}$ \\
\hline Chicken manure & $7.448 \mathrm{ab}$ & $106.7 \mathrm{ab}$ & $794.9 \mathrm{ab}$ & $6.68 \mathrm{ab}$ & $7.38 \mathrm{~b}$ & $106.3 \mathrm{~b}$ & $784.8 \mathrm{~b}$ & $6.59 \mathrm{~b}$ \\
\hline Pressed Olive Cake & $6.249 \mathrm{c}$ & $102.1 \mathrm{~b}$ & $636.9 \mathrm{c}$ & $5.35 \mathrm{c}$ & $6.23 \mathrm{c}$ & $102.5 \mathrm{c}$ & $638.3 \mathrm{c}$ & $5.36 \mathrm{c}$ \\
\hline Compost & $6.754 \mathrm{bc}$ & $107.1 \mathrm{ab}$ & $723.6 \mathrm{bc}$ & $6.08 \mathrm{bc}$ & $6.80 \mathrm{bc}$ & $107.0 \mathrm{~b}$ & $728.5 \mathrm{~b}$ & $6.12 \mathrm{~b}$ \\
\hline \multicolumn{9}{|l|}{ Bio fertilizers } \\
\hline Without Bio & $7.045 \mathrm{a}$ & 107.9 a & $760.7 \mathrm{a}$ & $6.39 \mathrm{a}$ & $7.03 \mathrm{a}$ & $107.5 \mathrm{a}$ & $756.8 \mathrm{a}$ & $6.36 \mathrm{a}$ \\
\hline Bio-1 & $6.978 \mathrm{a}$ & $108.6 \mathrm{a}$ & $760.6 \mathrm{a}$ & $6.39 \mathrm{a}$ & $7.01 \mathrm{a}$ & 108.9 a & $765.4 \mathrm{a}$ & $6.43 \mathrm{a}$ \\
\hline Bio- 2 & $7.459 \mathrm{a}$ & $103.8 \mathrm{a}$ & $776.7 \mathrm{a}$ & $6.52 \mathrm{a}$ & $7.41 \mathrm{a}$ & $104.1 \mathrm{a}$ & 773.9 a & $6.50 \mathrm{a}$ \\
\hline \multicolumn{9}{|c|}{ Organic fertilizers $x$ Bio fertilizers } \\
\hline Cow manure $x$ Without Bio & $7.62 \mathrm{bc}$ & $111.4 \mathrm{ab}$ & $850.3 \mathrm{bc}$ & $7.140 \mathrm{bc}$ & $7.56 \mathrm{bc}$ & $110.3 \mathrm{~b}$ & $833.9 \mathrm{~b}$ & $7.0 \mathrm{~b}$ \\
\hline Cow manure x Bio-1 & $7.90 \mathrm{~b}$ & $115.8 \mathrm{a}$ & $910.9 \mathrm{ab}$ & $7.65 \mathrm{ab}$ & $7.81 \mathrm{~b}$ & $116.8 \mathrm{a}$ & $910.2 \mathrm{a}$ & $7.65 \mathrm{a}$ \\
\hline Cow manure x Bio-2 & $9.06 \mathrm{a}$ & $106.3 \mathrm{ab}$ & $964.1 \mathrm{a}$ & $8.10 \mathrm{a}$ & $9.16 \mathrm{a}$ & $107.5 \mathrm{bc}$ & $986.0 \mathrm{a}$ & $8.28 \mathrm{a}$ \\
\hline Chicken manure $\mathrm{x}$ Without Bio & $7.09 \mathrm{~cd}$ & $106.1 \mathrm{ab}$ & $752.0 \mathrm{c}-\mathrm{e}$ & $6.32 \mathrm{c}-\mathrm{e}$ & $7.04 \mathrm{c}$ & $106.2 \mathrm{~b}-\mathrm{d}$ & $747.9 \mathrm{~cd}$ & $6.28 \mathrm{~cd}$ \\
\hline Chicken manure $x$ Bio-1 & $7.48 \mathrm{bc}$ & $108.9 \mathrm{ab}$ & $814.1 \mathrm{bc}$ & $6.837 \mathrm{bc}$ & $7.41 \mathrm{bc}$ & $107.3 \mathrm{bc}$ & $794.6 \mathrm{~b}-\mathrm{d}$ & $6.67 \mathrm{~b}-\mathrm{d}$ \\
\hline Chicken manure $x$ Bio-2 & $7.78 \mathrm{bc}$ & $105.1 \mathrm{ab}$ & $818.4 \mathrm{bc}$ & $6.873 \mathrm{bc}$ & $7.70 \mathrm{~b}$ & $105.3 \mathrm{~b}-\mathrm{d}$ & $811.8 \mathrm{bc}$ & $6.82 \mathrm{bc}$ \\
\hline Pressed Olive Cake x Without Bio & $5.94 \mathrm{e}$ & $106.8 \mathrm{ab}$ & $633.6 \mathrm{f}$ & $5.320 \mathrm{f}$ & $5.96 \mathrm{e}$ & $106.3 \mathrm{~b}-\mathrm{d}$ & $632.8 \mathrm{f}$ & $5.32 \mathrm{f}$ \\
\hline Pressed Olive Cake x Bio-1 & $6.17 \mathrm{e}$ & $100.2 \mathrm{~b}$ & $617.4 \mathrm{f}$ & $5.183 \mathrm{f}$ & $6.30 \mathrm{de}$ & $101.4 \mathrm{de}$ & 638.7 ef & 5.36 ef \\
\hline Pressed Olive Cake x Bio-2 & $6.64 \mathrm{de}$ & $99.42 \mathrm{~b}$ & 659.8 ef & $5.543 \mathrm{ef}$ & $6.44 \mathrm{de}$ & $99.93 \mathrm{e}$ & $643.4 \mathrm{ef}$ & $5.41 \mathrm{ef}$ \\
\hline Compost x Without Bio & $7.53 \mathrm{bc}$ & $107.1 \mathrm{ab}$ & $806.7 \mathrm{~b}-\mathrm{d}$ & $6.777 \mathrm{~b}-\mathrm{d}$ & $7.56 \mathrm{bc}$ & $107.3 \mathrm{bc}$ & $812.6 \mathrm{bc}$ & $6.83 \mathrm{bc}$ \\
\hline Compost x Bio-1 & $6.37 \mathrm{e}$ & $109.7 \mathrm{ab}$ & $699.8 \mathrm{~d}-\mathrm{f}$ & $5.880 \mathrm{~d}-\mathrm{f}$ & $6.52 \mathrm{~d}$ & $110.1 \mathrm{~b}$ & $718.2 \mathrm{de}$ & $6.03 \mathrm{de}$ \\
\hline Compost x Bio-2 & $6.36 \mathrm{e}$ & $104.4 \mathrm{ab}$ & 664.4 ef & $5.580 \mathrm{ef}$ & $6.32 \mathrm{de}$ & $103.6 \mathrm{c}-\mathrm{e}$ & $654.5 \mathrm{ef}$ & $5.50 \mathrm{ef}$ \\
\hline
\end{tabular}
* Means followed by the same alphabetical latter(s) within each column are not significantly different at $5 \%$
level, according to Duncan multiple range Test. 
concluded that the vigor growth plants were resulted due to application cow manure treatment, which is known that, its organic fertilizer addition to the soil resulted in slow release of nutrients along the plant life and improves soil chemical properties as well as maintaining soil fertility, consequently their absorption increase. Also, it is worth to mention that, good effect of organic nitrogen treatment would be to increasing plant growth parameters (Saleh et al., 2007; on tomato, El-Kafrawy and Radwan, 2008 on cucumber).

\section{Effect of bio fertilizers}

Results in Table 8 clear no significant differences on yield and its components by bio-fertilizer.

\section{Effect of interaction between organic fertilizers and Bio fertilizers}

Results in Table 8 show significant differences among organic fertilizers for early yield/fed. The interaction between organic and bio-fertilizers resulted in significant differences in case of early yield/fed., and the percentage of early yield to total yield.

Also, results in the same Table show significant effects for the interaction among organic fertilizer sources and bio-fertilizers. Cow manure $x$ Bio-1 treatment gave the highest value in case of average fruit weight / plant, while, Cow manure $\mathrm{x}$ Bio-2 treatment gave the superior values in cases of number of fruits/plant, yield /plant and yield/fed., in both seasons.

The increases in total fruits yield and its components might be attributed to the increase in vegetative growth characteristics (Tables 5 and 6) and reproductive phases of plant which have impact on total fruits yield and its components (Hamed, 1997; Turemis et al., 1998; Awad, 2005; Awad et al., 2006; Rathore et al., 2008).

However, this increase in yield and its components may be due to the increasing in vegetative growth parameters; i.e.; plant length, dry weight/plant, number of leaves as well as leaf area/plant as shown in Tables 5 and 6. It may be also stated that the sufficient addition and the efficient absorption of nutrients (NPK) were coupled together to promote the production for good yield and its components (Floresen et al., 1991; Ali, 2002).

\section{Fruit quality}

\section{Effect of organic fertilizers}

Results in Table 9 show significant differences among most of organic fertilizer sources. Fruit TSS (\%) had higher significant values in fruits of plants fertilized with chicken manure, cow manure and compost, while pressed olive cake produced the lowest fruit $\mathrm{TSS}(\%)$ in both seasons. Organic fertilizers are claimed to produce higher nutritional quality of vegetable fruits in forms of TSS (\%) in tomatoes (El-Kassas and Abd El-Mowly, 1999; Youssef et al., 2001; Bayoumi, 2005). Cow manure without bio addition resulted in the highest values of fruit firmness in both seasons, while compost without bio addition produced the lowest firmness values. These results are on agreement with Pelaez et al. (1984) on squash and Ali (2002) on cucumber Cow and chicken manures produced the highest fruit length values.

\section{Effect of bio fertilizers}

Results in Table 9 show no significant effects for biofertilizers on all determined parameters in both seasons.

\section{Effect of interaction between organic fertilizers and bio fertilizers}

Results in Table 9 show that the interaction between organic and bio fertilizers resulted in significant differences for all determined parameters in both seasons. Significant differences in TSS(\%) were appeared as a result of the interaction between organic and bio fertilizers, chicken 
Table (9): Effect of organic fertilizers, bio-fertilizers and their interactions on fruit quality of summer squash during 2013 and 2014 seasons.

\begin{tabular}{|c|c|c|c|c|c|c|c|c|}
\hline Character & $\begin{array}{c}\text { Fruit } \\
\text { TSS } \\
(\%) \\
\end{array}$ & $\begin{array}{c}\text { Fruit } \\
\text { Diameter } \\
(\mathrm{cm})\end{array}$ & $\begin{array}{c}\text { Fruit } \\
\text { Length } \\
\text { (cm) }\end{array}$ & $\begin{array}{c}\text { Fruit } \\
\text { Firmness } \\
\left(\mathrm{kg} / \mathrm{cm}^{2}\right)\end{array}$ & $\begin{array}{c}\text { Fruit } \\
\text { TSS } \\
(\%) \\
\end{array}$ & $\begin{array}{c}\text { Fruit } \\
\text { Diameter } \\
(\mathrm{cm})\end{array}$ & $\begin{array}{c}\text { Fruit } \\
\text { Length } \\
\text { (cm) }\end{array}$ & $\begin{array}{c}\text { Fruit } \\
\text { Firmness } \\
\left(\mathrm{kg} / \mathrm{cm}^{2}\right)\end{array}$ \\
\hline Treatment & \multicolumn{4}{|c|}{ first Season (2013) } & \multicolumn{4}{|c|}{ Second Season(2014) } \\
\hline \multicolumn{9}{|l|}{ Organic fertilizers } \\
\hline Cow manure & $5.04 \mathrm{ab}$ & $3.70 \mathrm{a}$ & $14.26 \mathrm{a}$ & $3.73 \mathrm{a}$ & $5.04 \mathrm{a}$ & $3.72 \mathrm{a}$ & $14.42 \mathrm{a}$ & $3.69 \mathrm{ab}$ \\
\hline Chicken manure & $5.06 \mathrm{a}$ & $3.73 \mathrm{a}$ & $14.31 \mathrm{a}$ & $3.76 \mathrm{a}$ & $5.07 \mathrm{a}$ & $3.72 \mathrm{a}$ & $14.22 \mathrm{a}$ & $3.75 \mathrm{a}$ \\
\hline Pressed Olive Cake & $4.96 \mathrm{~b}$ & $3.63 \mathrm{a}$ & $12.68 \mathrm{~b}$ & $3.53 \mathrm{ab}$ & $4.95 \mathrm{~b}$ & $3.54 \mathrm{a}$ & $12.57 \mathrm{~b}$ & $3.54 \mathrm{bc}$ \\
\hline Compost & $5.01 \mathrm{ab}$ & $3.46 \mathrm{a}$ & $12.88 \mathrm{ab}$ & $3.46 \mathrm{~b}$ & $5.0 \mathrm{ab}$ & $3.46 \mathrm{a}$ & $12.96 \mathrm{~b}$ & $3.45 \mathrm{c}$ \\
\hline \multicolumn{9}{|l|}{ Bio fertilizers } \\
\hline Without Bio & $5.03 \mathrm{a}$ & $3.65 \mathrm{a}$ & $13.61 \mathrm{a}$ & $3.68 \mathrm{a}$ & $5.03 \mathrm{a}$ & $3.60 \mathrm{a}$ & $13.61 \mathrm{a}$ & $3.68 \mathrm{a}$ \\
\hline Bio-1 & $5.01 \mathrm{a}$ & $3.54 \mathrm{a}$ & $13.57 \mathrm{a}$ & $3.59 \mathrm{a}$ & $5.02 \mathrm{a}$ & $3.53 \mathrm{a}$ & $13.56 \mathrm{a}$ & $3.56 \mathrm{a}$ \\
\hline Bio- 2 & $5.0 \mathrm{a}$ & $3.71 \mathrm{a}$ & $13.42 \mathrm{a}$ & $3.58 \mathrm{a}$ & $5.00 \mathrm{~b}$ & $3.71 \mathrm{a}$ & $13.46 \mathrm{a}$ & $3.58 \mathrm{a}$ \\
\hline \multicolumn{9}{|l|}{ Organic fertilizers $x$ Bio fertilizers } \\
\hline Cow manure x Without Bio & $5.07 \mathrm{ab}$ & $3.70 \mathrm{ab}$ & $14.39 \mathrm{ab}$ & $3.86 \mathrm{a}$ & $5.07 \mathrm{~b}$ & $3.71 \mathrm{bc}$ & $14.48 \mathrm{a}$ & $3.85 \mathrm{a}$ \\
\hline Cow manure $x$ Bio-1 & $5.02 \mathrm{a}-\mathrm{e}$ & $3.68 \mathrm{ab}$ & $14.04 \mathrm{ab}$ & $3.68 \mathrm{a}-\mathrm{d}$ & $5.03 \mathrm{c}$ & $3.71 \mathrm{bc}$ & $14.18 \mathrm{a}$ & $3.61 \mathrm{bc}$ \\
\hline Cow manure $x$ Bio-2 & $5.01 \mathrm{~b}-\mathrm{f}$ & $3.71 \mathrm{ab}$ & $14.36 \mathrm{ab}$ & 3.65 a-e & $5.01 \mathrm{~d}$ & $3.75 \mathrm{~b}$ & $14.59 \mathrm{a}$ & $3.60 \mathrm{bc}$ \\
\hline Chicken manure $x$ Without Bio & $5.06 \mathrm{a}-\mathrm{c}$ & $3.78 \mathrm{ab}$ & $14.56 \mathrm{a}$ & $3.75 \mathrm{ab}$ & $5.07 \mathrm{~b}$ & $3.76 \mathrm{~b}$ & $14.49 \mathrm{a}$ & $3.77 \mathrm{ab}$ \\
\hline Chicken manure x Bio-1 & $5.08 \mathrm{a}$ & $3.80 \mathrm{ab}$ & $14.62 \mathrm{a}$ & $3.72 \mathrm{a}-\mathrm{c}$ & $5.09 \mathrm{a}$ & $3.77 \mathrm{~b}$ & $14.49 \mathrm{a}$ & $3.71 \mathrm{ab}$ \\
\hline Chicken manure x Bio-2 & $5.04 \mathrm{a}-\mathrm{d}$ & $3.62 \mathrm{~b}$ & $13.74 \mathrm{bc}$ & $3.81 \mathrm{ab}$ & $5.04 \mathrm{c}$ & $3.63 \mathrm{~b}-\mathrm{d}$ & $13.69 \mathrm{~b}$ & $3.78 \mathrm{ab}$ \\
\hline Pressed Olive Cake x Without Bio & $4.98 \mathrm{e}-\mathrm{g}$ & $3.69 \mathrm{ab}$ & $12.86 \mathrm{de}$ & $3.73 \mathrm{ab}$ & $4.96 \mathrm{e}$ & $3.50 \mathrm{~cd}$ & $12.75 \mathrm{c}$ & $3.72 \mathrm{ab}$ \\
\hline Pressed Olive Cake x Bio-1 & $4.95 \mathrm{fg}$ & $3.11 \mathrm{c}$ & $12.32 \mathrm{e}$ & $3.40 \mathrm{e}$ & $4.95 \mathrm{ef}$ & $3.09 \mathrm{e}$ & $12.27 \mathrm{~d}$ & $3.43 \mathrm{~cd}$ \\
\hline Pressed Olive Cake x Bio-2 & $4,94 \mathrm{~g}$ & $4.08 \mathrm{a}$ & $12.86 \mathrm{de}$ & $3.45 \mathrm{c}-\mathrm{e}$ & $4.94 \mathrm{f}$ & $4.02 \mathrm{a}$ & $12.70 \mathrm{c}$ & $3.46 \mathrm{~cd}$ \\
\hline Compost x Without Bio & $5.01 \mathrm{c}-\mathrm{f}$ & $3.41 \mathrm{bc}$ & $12.62 \mathrm{de}$ & $3.39 \mathrm{e}$ & $5.01 \mathrm{~d}$ & $3.41 \mathrm{~d}$ & $12.73 \mathrm{c}$ & $3.36 \mathrm{~d}$ \\
\hline Compost x Bio-1 & $5.0 \mathrm{~d}-\mathrm{g}$ & $3.56 \mathrm{~b}$ & $13.29 \mathrm{~cd}$ & $3.57 \mathrm{~b}-\mathrm{e}$ & $5.0 \mathrm{~d}$ & $3.54 \mathrm{~b}-\mathrm{d}$ & $13.31 \mathrm{~b}$ & $3.50 \mathrm{~cd}$ \\
\hline Compost x Bio-2 & $5.01 \mathrm{~b}-\mathrm{f}$ & $3.40 \mathrm{bc}$ & $12.74 \mathrm{de}$ & $3.41 \mathrm{de}$ & $5.0 \mathrm{~d}$ & $3.42 \mathrm{~d}$ & $12.85 \mathrm{c}$ & $3.48 \mathrm{~cd}$ \\
\hline
\end{tabular}

* Means followed by the same alphabetical latter(s) within each column are not significantly different at 5\% level, according to Duncan multiple range Test. 
manure and Bio-1 produced the highest value of TSS (\%). The lowest TSS (\%) value resulted from the interaction between pressed olive cake and Bio-2 in both seasons. Many Researchers found that squash plants treated with pressed olive cake and Bio- 2 produced the highest value in fruit diameter, except for chicken manure + Bio-2.

On the other hand, Such increase in TSS, fruit dimensions and firmness could be attributed to cow manure which contained a lot of macro and micro elements, carbohydrates and growth hormones, vitamins and amino acids and their involvement in one or more of important biological functions which referred to the simulate plant growth and consequently increased fruit quality contents (Eris et al., 1995).

The highest fruit diameter was recorded with application of pressed olive cake $\mathrm{x}$ Bio-2 in both seasons. The highest fruit length was recorded with application of cow manure $\mathrm{x}$ without Bio in both seasons.

\section{Chemical Contents}

\section{Effect of organic fertilizers}

Results in Tables 10 and 11 clear that there were significant effects for organic fertilizers on leaves content of $\mathrm{P}$ and $\mathrm{K}$ in the first season and $\mathrm{N}$ and $\mathrm{P}$ in the second season. On the other hand, there were significant effects for fruits content of $\mathrm{P}$ in the first and second seasons.

\section{Effect of bio fertilizers}

Results in Tables 10 and 11 clear that there were no significant differences among bio-fertilizer treatments on NPK contents of squash leaves and fruits.

\section{Effect of interaction between organic fertilizers and Bio fertilizers}

Tables 10 and 11 clear that there were significant effects among the interaction treatments in both seasons, except $\mathrm{N}$ and $\mathrm{K}$ contents of fruits in the first season and $\mathrm{P}$ and $\mathrm{K}$ contents of leaves in the second season. The highest contents in leaves were recorded for $\mathrm{N}$ when cow manure was mixed with Bio-1 (2.323\%) in the first season and with Bio-2 (2.13) in the second one; for $\mathrm{P}$ with chicken manure and Bio-2 (0.137); for $\mathrm{K}$ with compost without bio (1.10) in the first season.

The highest contents in fruits were recorded for $\mathrm{N}(2.45 \%)$ with application of pressed olive cake and bio- 2 in the first season; for $\mathrm{P}(0.333 \%$ and $0.280 \%$ in the first and second seasons, respectively) with application of cow manure with Bio-1 and compost without bio, respectively; for $\mathrm{K}$ (0.287) with application of pressed olive cake and bio- 1 in the second season. The increments of $\mathrm{N}, \mathrm{P}$ and $\mathrm{K}$ contents in the leaves and fruits might be attributed to organic elements constituents as reported by Hamed (1997) and El-Aidy et al. (2002). Shaban, Sally (2009) found that percent N,P and $\mathrm{K}$ in the leaves and fruits of summer squash were significantly influenced by different types of organic nitrogen fertilizer sources.Also, Shehata (2001) on squash and El-Sherif (2006) on cucumber found that application of organic fertilizers increased the concentration of $\mathrm{N}, \mathrm{P}$ and $\mathrm{K}$ in both shoots and fruits.

On the other hand, many researchers reported that the steady release of nitrogen from organic manures in form of ammonium at relatively slow release probably caused low nitrate contents in the fruits of squash plants (Clark et al., 1999;

\section{Abd El-Kawy, 2003; Awad,2005; Ibrahim and Selim, 2007).}

In addition, El-Sherif (2006) indicated that increment uptake of $\mathrm{N}, \mathrm{P}$ and $\mathrm{K}$ in the leaves and fruits may be due to higher availability of the nutrients which resulted in better root growth and increased physiological activity of root to absorb the nutrients through decomposition of organic matter that led to increase their concentration in plant leaves and fruits. 
Table (10): Effect of organic fertilizers, bio-fertilizers and their interactions on chemical contents in leaves and fruits of summer squash plants during 2013 season.

\begin{tabular}{|c|c|c|c|c|c|c|}
\hline \multirow[b]{2}{*}{ Treatment } & \multicolumn{3}{|c|}{ Leaves } & \multirow[b]{2}{*}{$\mathbf{N}(\%)$} & \multirow[b]{2}{*}{$\mathbf{P}(\%)$} & \multirow{2}{*}{$\begin{array}{c}\text { Fruits } \\
\mathbf{K}(\%)\end{array}$} \\
\hline & $\mathbf{N}(\%)$ & $\mathbf{P}(\%)$ & $\mathbf{K}(\%)$ & & & \\
\hline \multicolumn{7}{|l|}{ Organic fertilizers } \\
\hline Cow manure & $2.804 \mathrm{a}$ & $0.100 \mathrm{~b}$ & $0.626 \mathrm{~b}$ & $2.11 \mathrm{a}$ & $0.312 \mathrm{a}$ & $0.137 \mathrm{a}$ \\
\hline Chicken manure & $2.713 \mathrm{a}$ & $0.103 \mathrm{ab}$ & $0.653 \mathrm{ab}$ & $1.99 \mathrm{a}$ & $0.282 \mathrm{ab}$ & $0.111 \mathrm{a}$ \\
\hline Pressed Olive Cake & $2.534 \mathrm{a}$ & $0.117 \mathrm{ab}$ & $0.912 \mathrm{a}$ & $1.92 \mathrm{a}$ & $0.222 \mathrm{~b}$ & $0.140 \mathrm{a}$ \\
\hline Compost & $2.356 \mathrm{a}$ & $0.121 \mathrm{a}$ & $0.916 \mathrm{a}$ & $1.77 \mathrm{a}$ & $0.240 \mathrm{~b}$ & $0.154 \mathrm{a}$ \\
\hline \multicolumn{7}{|l|}{ Bio fertilizers } \\
\hline Without Bio & $2.664 \mathrm{a}$ & $0.106 \mathrm{a}$ & $0.774 \mathrm{a}$ & $2.01 \mathrm{a}$ & $0.277 \mathrm{a}$ & $0.139 \mathrm{a}$ \\
\hline Bio-1 & $2.638 \mathrm{a}$ & $0.112 \mathrm{a}$ & $0.800 \mathrm{a}$ & $1.87 \mathrm{a}$ & $0.248 \mathrm{a}$ & $0.133 \mathrm{a}$ \\
\hline Bio- 2 & $2.504 \mathrm{a}$ & $0.113 \mathrm{a}$ & $0.756 \mathrm{a}$ & $1.97 \mathrm{a}$ & $0.268 \mathrm{a}$ & $0.134 \mathrm{a}$ \\
\hline \multicolumn{7}{|l|}{ Organic fertilizers $x$ bio fertilizers } \\
\hline Cow manure $x$ Without Bio & $2.953 \mathrm{ab}$ & $0.120 \mathrm{ab}$ & $0.527 \mathrm{e}$ & $2.21 \mathrm{a}$ & $0.337 \mathrm{a}$ & $0.150 \mathrm{a}$ \\
\hline Cow manure x Bio-1 & $3.137 \mathrm{a}$ & $0.100 \mathrm{ab}$ & $0.650 \mathrm{de}$ & $2.11 \mathrm{a}$ & $0.267 \mathrm{~b}-\mathrm{d}$ & $0.133 \mathrm{a}$ \\
\hline Cow manure x Bio-2 & $2.323 \mathrm{~b}$ & $0.080 \mathrm{ab}$ & $0.700 \mathrm{c}-\mathrm{e}$ & $2.00 \mathrm{a}$ & $0.333 \mathrm{a}$ & $0.127 \mathrm{a}$ \\
\hline Chicken manure $x$ Without Bio & $2.947 \mathrm{ab}$ & $0.073 \mathrm{~b}$ & $0.650 \mathrm{de}$ & $1.86 \mathrm{a}$ & $0.297 \mathrm{ab}$ & $0.117 \mathrm{a}$ \\
\hline Chicken manure x Bio-1 & $2.477 \mathrm{ab}$ & $0.100 \mathrm{ab}$ & $0.650 \mathrm{de}$ & $1.84 \mathrm{a}$ & $0.263 \mathrm{~b}-\mathrm{d}$ & $0.110 \mathrm{a}$ \\
\hline Chicken manure $x$ Bio-2 & $2.717 \mathrm{ab}$ & $0.137 \mathrm{a}$ & $0.660 \mathrm{de}$ & $2.28 \mathrm{a}$ & $0.287 \mathrm{a}-\mathrm{c}$ & $0.107 \mathrm{a}$ \\
\hline Pressed Olive Cake x Without Bio & $2.397 \mathrm{ab}$ & $0.110 \mathrm{ab}$ & $0.820 \mathrm{~b}-\mathrm{d}$ & $2.19 \mathrm{a}$ & $0.223 \mathrm{~d}$ & $0.147 \mathrm{a}$ \\
\hline Pressed Olive Cake x Bio-1 & $2.597 \mathrm{ab}$ & $0.123 \mathrm{ab}$ & $0.917 \mathrm{a}-\mathrm{c}$ & $1.77 \mathrm{a}$ & $0.213 \mathrm{~d}$ & $0.130 \mathrm{a}$ \\
\hline Pressed Olive Cake x Bio-2 & $2.610 \mathrm{ab}$ & $0.117 \mathrm{ab}$ & $1.000 \mathrm{ab}$ & $1.79 \mathrm{a}$ & $0.230 \mathrm{~cd}$ & $0.143 \mathrm{a}$ \\
\hline Compost $\mathrm{x}$ Without Bio & $2.360 \mathrm{~b}$ & $0.120 \mathrm{ab}$ & $1.100 \mathrm{a}$ & $1.78 \mathrm{a}$ & $0.250 \mathrm{~b}-\mathrm{d}$ & $0.143 \mathrm{a}$ \\
\hline Compost x Bio-1 & $2.340 \mathrm{~b}$ & $0.123 \mathrm{ab}$ & $0.983 \mathrm{ab}$ & $1.75 \mathrm{a}$ & $0.247 \mathrm{~b}-\mathrm{d}$ & $0.160 \mathrm{a}$ \\
\hline Compost x Bio-2 & $2.367 \mathrm{~b}$ & $0.120 \mathrm{ab}$ & $0.663 \mathrm{de}$ & $1.81 \mathrm{a}$ & $0.223 \mathrm{~d}$ & $0.160 \mathrm{a}$ \\
\hline
\end{tabular}

* Means followed by the same alphabetical latter (s) within each column are not significantly different at $5 \%$ level, according to Duncan multiple range Test. 
Table (11): Effect of organic fertilizers, bio-fertilizers and their interactions on chemical contents in leaves and fruits of summer squashplants during 2014season

\begin{tabular}{|c|c|c|c|c|c|c|}
\hline \multirow[b]{2}{*}{ Treatment } & \multicolumn{3}{|c|}{ Leaves } & \multicolumn{3}{|r|}{ Fruits } \\
\hline & $\mathbf{N}(\%)$ & $\mathbf{P}(\%)$ & $\mathbf{K}(\%)$ & $\mathbf{N}(\%)$ & $\mathbf{P}(\%)$ & $\mathbf{K}(\%)$ \\
\hline \multicolumn{7}{|l|}{ Organic fertilizers } \\
\hline Cow manure & $1.87 \mathrm{a}$ & $0.083 \mathrm{a}$ & $0.512 \mathrm{a}$ & $1.68 \mathrm{a}$ & $0.202 \mathrm{c}$ & $0.182 \mathrm{a}$ \\
\hline Chicken manure & $1.30 \mathrm{~b}$ & $0.074 \mathrm{ab}$ & $0.500 \mathrm{a}$ & $1.65 \mathrm{a}$ & $0.226 \mathrm{~b}$ & $0.199 \mathrm{a}$ \\
\hline Pressed Olive Cake & $1.68 \mathrm{ab}$ & $0.060 \mathrm{~b}$ & $0.553 \mathrm{a}$ & $2.02 \mathrm{a}$ & $0.251 \mathrm{a}$ & $0.248 \mathrm{a}$ \\
\hline Compost & $1.64 \mathrm{ab}$ & $0.070 \mathrm{ab}$ & $0.527 \mathrm{a}$ & $1.79 \mathrm{a}$ & $0.249 \mathrm{a}$ & $0.196 \mathrm{a}$ \\
\hline \multicolumn{7}{|l|}{ Bio fertilizers } \\
\hline Without Bio & $1.63 \mathrm{a}$ & $0.076 \mathrm{a}$ & $0.548 \mathrm{a}$ & $1.65 \mathrm{a}$ & $0.235 \mathrm{a}$ & $0.188 \mathrm{a}$ \\
\hline Bio-1 & $1.59 \mathrm{a}$ & $0.071 \mathrm{a}$ & $0.503 \mathrm{a}$ & $1.87 \mathrm{a}$ & $0.232 \mathrm{a}$ & $0.219 \mathrm{a}$ \\
\hline Bio- 2 & $1.65 \mathrm{a}$ & $0.069 \mathrm{a}$ & $0.518 \mathrm{a}$ & $1.84 \mathrm{a}$ & $0.229 \mathrm{a}$ & $0.211 \mathrm{a}$ \\
\hline \multicolumn{7}{|l|}{ Organic fertilizers $x$ bio fertilizers } \\
\hline Cow manure $x$ Without Bio & $1.78 \mathrm{ab}$ & $0.090 \mathrm{a}$ & $0.537 \mathrm{a}$ & $1.89 \mathrm{a}-\mathrm{e}$ & $0.227 \mathrm{a}-\mathrm{c}$ & $0.160 \mathrm{c}$ \\
\hline Cow manure x Bio-1 & $1.71 \mathrm{ab}$ & $0.080 \mathrm{a}$ & $0.467 \mathrm{a}$ & $1.64 \mathrm{~b}-\mathrm{e}$ & $0.197 \mathrm{c}$ & $0.173 \mathrm{c}$ \\
\hline Cow manure x Bio-2 & $2.13 \mathrm{a}$ & $0.080 \mathrm{a}$ & $0.533 \mathrm{a}$ & $1.52 \mathrm{c}-\mathrm{e}$ & $0.183 \mathrm{c}$ & $0.213 \mathrm{bc}$ \\
\hline Chicken manure $x$ Without Bio & $1.43 \mathrm{ab}$ & $0.077 \mathrm{a}$ & $0.517 \mathrm{a}$ & $1.42 \mathrm{de}$ & $0.210 \mathrm{bc}$ & $0.183 \mathrm{c}$ \\
\hline Chicken manure x Bio-1 & $1.13 \mathrm{~b}$ & $0.077 \mathrm{a}$ & $0.487 \mathrm{a}$ & $1.52 \mathrm{c}-\mathrm{e}$ & $0.227 \mathrm{a}-\mathrm{c}$ & $0.213 \mathrm{bc}$ \\
\hline Chicken manure x Bio-2 & $1.33 \mathrm{~b}$ & $0.070 \mathrm{a}$ & $0.497 \mathrm{a}$ & $2.02 \mathrm{a}-\mathrm{d}$ & $0.240 \mathrm{a}-\mathrm{c}$ & $0.200 \mathrm{bc}$ \\
\hline Pressed Olive Cake x Without Bio & $1.51 \mathrm{ab}$ & $0.060 \mathrm{a}$ & $0.570 \mathrm{a}$ & $1.52 \mathrm{c}-\mathrm{e}$ & $0.223 \mathrm{a}-\mathrm{c}$ & $0.200 \mathrm{bc}$ \\
\hline Pressed Olive Cake x Bio-1 & $1.82 \mathrm{ab}$ & $0.063 \mathrm{a}$ & $0.583 \mathrm{a}$ & $2.10 \mathrm{a}-\mathrm{c}$ & $0.270 \mathrm{ab}$ & $0.287 \mathrm{a}$ \\
\hline Pressed Olive Cake x Bio-2 & $1.70 \mathrm{ab}$ & $0.057 \mathrm{a}$ & $0.507 \mathrm{a}$ & $2.45 \mathrm{a}$ & $0.260 \mathrm{ab}$ & $0.257 \mathrm{ab}$ \\
\hline Compost x Without Bio & $1.79 \mathrm{ab}$ & $0.077 \mathrm{a}$ & $0.567 \mathrm{a}$ & $1.79 \mathrm{~b}-\mathrm{e}$ & $0.280 \mathrm{a}$ & $0.210 \mathrm{bc}$ \\
\hline Compost x Bio-1 & $1.69 \mathrm{ab}$ & $0.063 \mathrm{a}$ & $0.477 \mathrm{a}$ & $2.22 \mathrm{ab}$ & $0.233 \mathrm{a}-\mathrm{c}$ & $0.203 \mathrm{bc}$ \\
\hline Compost $x$ Bio-2 & $1.45 \mathrm{ab}$ & $0.070 \mathrm{a}$ & $0.537 \mathrm{a}$ & $1.36 \mathrm{e}$ & $0.233 \mathrm{a}-\mathrm{c}$ & $0.173 \mathrm{c}$ \\
\hline
\end{tabular}

* Means followed by the same alphabetical latter(s) within each column are not significantly different at $5 \%$ level, according to Duncan multiple range Test. 


\section{REFERENCES}

Abd El-Fattah, M.A. and Sorial, M.E. (2000). Sex expression and productivity responses of summer squash to biofertilizers application under different nitrogen levels. Zagzig J. Agric. Res., 27: 255-281.

Abd El-Kawy, T.F. (2003). The effect of some organic and inorganic fertilizers on plant growth and fruits quality of cucumber plant under plastic houses. M.Sc. Thesis, Fac. Agric. Ain Shams Univ., Egypt.

Abo-Hadid, A.F. (2001). The current studies of organic horticulture production and marketing in Egypt. Sym. Int. Agric. Biolgique, Agadir, Morac, 7-10.

Agbede, T.M.; Ojeniyi, S.O. and Adeyemo, A.J. (2008). Effect of poultry manure on soil physical and chemical properties, growth and grain yield of sorghum in South West, Nigeria. American-Eurasian J. Sustainable Agric. 2: 72-77.

Aksoy, U. (2001). Ecological Farming. II. Ecological Farming Symposium in Turkey. 14-16 December, Antalya.

Ali, H.H. (2002). Studies on keeping quality and storability of cucumber fruit under organic farming system in greenhouses. M.Sc. Thesis, Fac. Agric. Cairo, Egypt.

Awad, El. M. M. (2005). The influence of organic and mineral fertilization on growth, yield and quality of potato crop. J. Agric. Sc. Mansoura Univ., 30 (12): 7965 - 7974.

Awad, El.M.M.; Youssef, N.S. and ElShell, Z.S. (2006). Effect of foliar spraying seaweed extracts on growth, yield and quality of potato crop. J. Agric. Sci. Mansoura Univ., 31 (10): 6549-6559.

Ayeni L.S.; Omole, T.O.; Adeleye, E.O. and Ojeniyi, S.O. (2010). Integrated application of poultry manure and NPK fertilizer on performance of tomato in derived savannah transition zone of South West Nigeria. Nat. and Sci., 8 (2): 50 - 54.

Bayoumi, Y.A. (2005). Studies on organic production of tomato crop. Ph.D. Thesis, Fac. Agric. Kafr EL-Sheikh, Tanta Univ. Egypt.

Bremner, J.M. and Mulvaney, C.S. (1982). Nitrogentotal. P.595-624. In. A.L. page (ed.), methods of soil analysis. Agron. No.9, part 2: chemical and microbiological properties $2^{\text {nd }}$ ed., $A M$. Soc. Agron., Madison, WI. USA.

Brown, J.D. and Lilliand, O. (1946). Rapid determination of potassium and soudium in plant material and soil extracts by flam photometry, Proc. Ame. Soc. Hort. Sci., 48:341-346.

Chaturvedi, S.; Kumar, A.; Singh, B.; Nain, L.; Joshi, M. and Satya, S. (2012). Bioaugmented composting of Jatrophade-oiled cake and vegetable waste under aerobic and partial anaerobic conditions. J. Basic Microbio. (In Press).

Clark, M.S.; W.R. Horwath; Shennan, C.; Scow, K.M.; Lantani, W.T. and Ferris, H. (1999). Nitrogen, weeds and water as yield limiting factors in conventional, low- input and organic tomato systems. Agric. Ecosyst. and Environ., 73:257-270.

Clement, R. and Bernal, M.P. (2006). Fractionation of heavy metals and distribution of organic carbon in two contaminated soils amended with humic substances. Chemosphere, 64: 1264-1273.

Darwish, Faysa M. (2002). Effect of different fertilizer source and levels on growth, yield and quality of tomato. Ph.D. Thesis, Fac. Agric. Cairo Univ., Egypt.

Duncan, D. B (1958). Multiple Range and Multiple F Test. Biometrics 11:1-42. 
SINAI Journal of Applied Sciences (ISSN: 2314-6079) Vol. (5) Is.:(1), Aug. 2016

El-Aidy, F.; El-zawily, A.I.; El-Sawy, B.I. and Hamed, E.M. (2002). Effect of seaweed extracts on sweet pepper plants grown under protected cultivation. $2^{\text {nd }}$ Int. Conf. Hort. Sci., Kafr El-Sheikh, Tanta Univ., Egypt, 28: (3/11): 10001012.

El-Ghanam, M.M.M.; Osman, A.S.A. and Kotb, K.I. (2005). Enrichment of some manufactured organic residues with mineral fertilizers in relation to wheat plants and electrical conductivity of sandy soil. J. Agric. Res., 30 (2): 775-789.

El-Kafrawy, A.A. and Radwan, E.A. (2008). Effect of different levels of compost on air and soil borne diseases, vegetative growth and yield of cucumber under protected cultivation. J.Agric. Sci. Mansoura Univ., 33 (3): 2165-2176.

El-Kassas, A.I. and Abd El-Mowly, S.E. (1999). Effect of some organic manures on growth and productivity of tomato plants grown in plastic green houses under North Sinai conditions. J. Agric. Mansoura Univ., 24 (12): 7645-7655.

El-Sherif, M.F.A. (2006). Growth and yield of cucumber as influenced by compost and nitrogen fertilizer in sand soil using nuclear technique for determination of nitrogen. $\mathrm{Ph}$. D. Thesis, Fac. Agric., Ain Shams Univ., Cairo, Egypt.

Eris, A.; Sivritepe, H.O. and Sivritepa, N. (1995). The effects of seaweed (Ascophyllum nodosum) extract on yield and quality criteria in peppers. Acta Hort., 412: 185-192.

Floresen, E.; Ciofu, R.; Vagialo, M.; Dumitru, M. and Berinde, A. (1991). The effect of maturing with urban-waste compost on the yield and quality of cucumbers grown in unheated green houses. Hort. Sci. 34 (1) 17-29 (CF Hort. Abst. 4498).

Galal, A.A.A.; Marzouk, N.M.; AlBassyuni, M.S.S. and Hassan, N.M.K.
(2012). Influence of organic nitrogen fertilizers replacement rates associated with Azosprillum spp., enrichment on tomato. J. Appl. Sci. Res., 9 (3): 19521959.

Hamed, E.M. (1997). Studies on seaweed extracts and shoot pruning on sweet peeper yield under plastic greenhouse. M.Sc. Thesis, Fac. Agric. Tanta Univ., Egypt.

Ibrahim, E.A. and Selim, E.M. (2007). Effect of irrigation intervals and nitrogen fertilizer rates on summer squash (Cucurbita pepo L.) growth, yield, nutritional status and water use efficiency. J. Agric. Sci., Mansoura Univ., 32 (12): 10333-10345.

Malak, A.E.R.; El-Bassiony, A.M. and Hoda, A.M. (2008). Behaviour of some micronutrients in soil and tomato plant organs under different levels and types of fertilizers. Austr., J. Basic and Appl. Sci., 2 (2): 288-295.

Marchner, H. (1995). Mineral Nutrition of Higher Plants. Academic Press Int, San Diego, CA, USA.

Pelaez, R.J.; Pave Calera, D.L. and Jaramillo, V.J. (1984). Response of squash (Cucurbita pepo L.) to fertilization with nitrogen, phosphorus, potassium and organic matter. Acta Agronomica, 34 (3): 86-95.

Piper, C.S. (1950). Soil and Plant Analysis. Int. Sci. Publishes, Inc., New York.

Ramakrishnan, $K$ and G. Selvakumar. (2012). Effect of biofertilizers on enhancement of growth and yield on tomato (Lycopersicum esculentum Mill.) Int., J. Res. in Botany, 2 (4): 20-23.

Rathore, S.S.; Chaudary, D.R.; Boricha, G.N.; Ghosh, A.; Zadope, S.T. and Patalia, J.S. (2008). Effect of seaweed extracts on growth, yield and nutrient uptake of soybean (Glycine max) under rainfed conditions. South Afr. J. Botany (11): 962-971 (Science direct Abst.). 
Reddy, T.Y. and Reddi, G.H.S. (2002). Principles Agron. $3^{\text {th }}$ Ed., Kalyani Publishers, India, 526.

Refai, E.F.S. and Mohamed, M.F. (2009). Population and single plant-derived inbred line analysis for sex expression in summer squash (Cucurbita pepo L.) cv. Eskandrani. Assuit Univ. Bull. Environ Res., $12: 1$.

Safia, M.; Adams, A.M.A. and Abdalla, M. (2001). The productivity of sweet pepper (Capsicum annuum L.) plant cultivated under plastic houses as affected by some organic maures as well as chemical fertilizer. Egypt. J. Appl. Sci., 16 (1): 18-27.

Saleh, M.M.; Darwish, F.M. and Hana, M.M. (2007). Effect of different types and levels of organic fertilizer combined with bio-fertilizer on growth and yield of tomato grown in sandy soil. J. Agric. Sci., Mansura Univ., 32 (7): 5553- 5568.

Sarhan, T.Z.; Ghurbtat, H.M. and Jiyan, A.T. (2011). Effect of bio and organic fertilizers on growth, yield and fruit quality of summer squash. Sarhad J. Agric., 27 (3):377-383.

Shaban, Sally M.S.A. (2009). Effect of organic farming on summer squash yield production. Ph. D Thesis, Agric. Sci. Fac. Agric., Mansoura Univ.,112.

Shehata, S.M. (2001). Effect of some organic wastes application on growth, chemical contents and yield of squash plant. J. Agric. Sci., Mansoura Univ., 26 (9): 5695-5704.

Turemis, N.F.; Kafkas, S. and Conickeiogla, N. (1998). Effect of fertilizer produced from the seaweed Ascophyllum nodosum on strawberry yield and quality. Hort. Abstr., 68 (12): 1376.

Yeussef, A.M.; El-Feuly, A.H.; Yeussef, M.S. and Mohamedion, S.A. (2001). Effect of using organic and chemical fertilizers in fustigation system on yield and fruits quality of tomato. Egypt. J. Hort., 28 (1): 59-77.

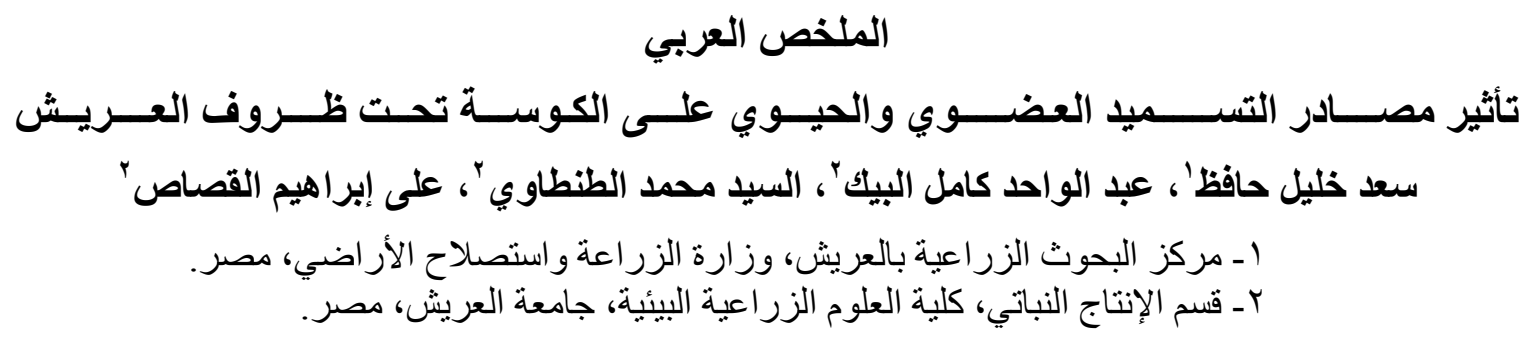

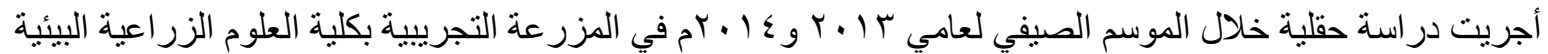

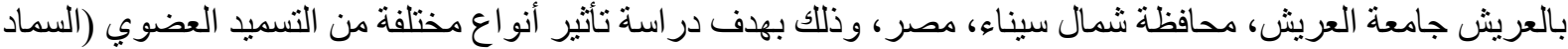

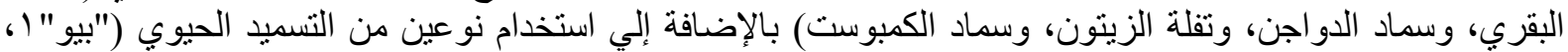

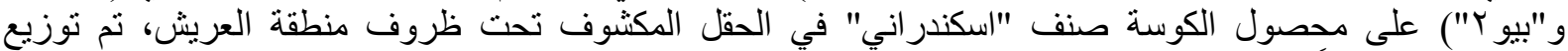

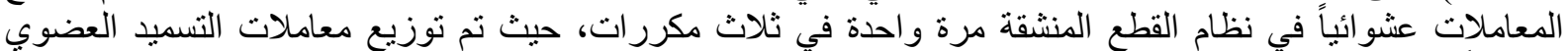

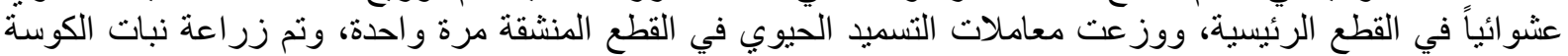

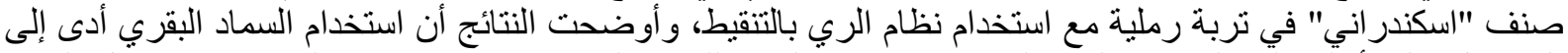

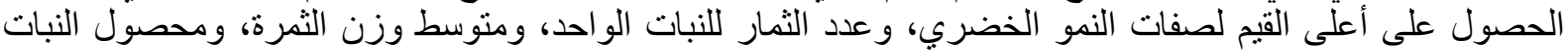

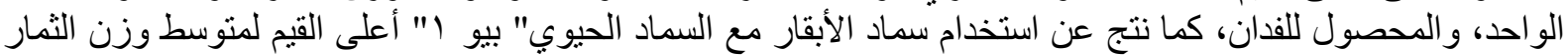

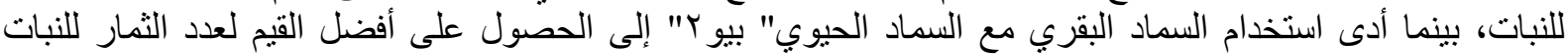

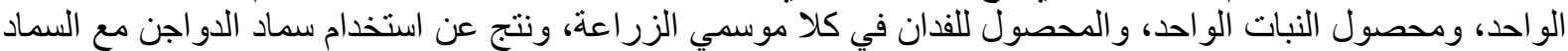
الحيوي " بيو ا" أعلى محتوى للثمار من المو اد الصلبة الذائبة الكلية.

الكلمات الاسترشادية: التسميد العضوي، الحيوي، السماد البقري، وسماد الدواجن، وتفله الزيتون، وسماد الكمبوست، الكوسة.

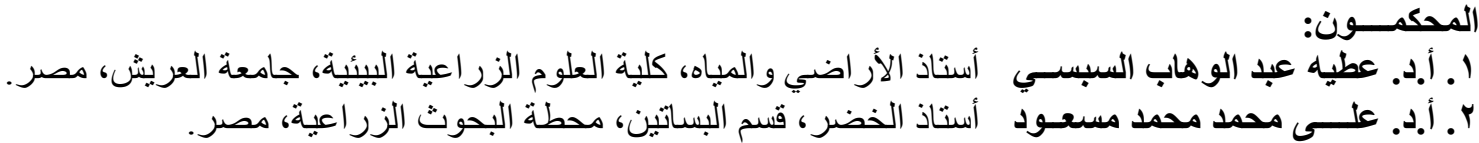

